\title{
Encuentro Internacional sobre Arquitectura y Urbanismo en América. Universidades UNICAMP y PUC de Campinas, São Paulo, Brasil. La arquitectura del Gran Caribe: Un acercamiento crítico
}

\author{
International Conference on Architecture and Urbanism in America. \\ UNICAMP and PUCCAMP, São Paulo, Brazil. \\ The architecture of the Wider Caribbean: a critical approach
}

$<$ Resumen>

El presente artículo nos propone una revisión y proyección del estado de situación de la arquitectura del Caribe. Un desarrollo heterogéneo, determinado por las particularidades que definen y construyen la experiencia arquitectónica de cada una de las naciones que conforman el gran Caribe y la conformación de sus ciudades. Un trabajo expuesto en el marco del encuentro internacional sobre arquitectura y urbanismo en América Latina, desarrollado en la Universidad Estadual de Campinas, Brasil, sede del próximo Seminario de Arquitectura Latinoamericana, SAL, a efectuarse el año 2011

$<$ Abstract $>$

This article proposes a review and projection of the status of caribbean architecture. A heterogeneous development determined by the details that define and build the architectural experience of each of the nations of the Wider Caribbean and the formation of their cities. This work was presented at the International Meeting on Architecture and Urbanism in Latin America, which took place at UNICAMP, Brazil, venue of the Seminar of Latin American Architecture, SAL, to be held in 201

<PALABRAS CLAVE>

ARQUITECTURA Y CRITICA / GRAN CARIBE / ARQUITECTURA CONTEMPORÁNEA

\section{$<K E Y W O R D S>$}

ARCHITECTURE AND CRITICISM \& WIDER CARIBBEAN \& CONTEMPORARY ARCHITECTURE
Abordar desde la crítica, la dispersa y extensa región del Gran Caribe ha sido y siempre será una tarea difícil y en gran medida, imposible. Es igual a querer delinear la cultura de las naciones vinculadas por el Mar Mediterráneo europeo con un sólo trazo, en un sólo lienzo; si bien la tarea es intelectualmente atractiva y ciertamente factible, años de esfuerzos infructuosos han demostrado lo contrario. Aunque tenemos la certeza de que algunos

\footnotetext{
Director principal de Moré Arquitectos, S.R.L. Graduado en la Universidad Nacional Pedro Henríquez Ureña (1979). El Arq. Moré posee una amplia experiencia profesional, incluyendo estudios de posgrado en las Universidades de Florencia (Italia); Harvard, Florida, National Gallery of Art en Washington (EUA) y en la Universidad Nacional Autónoma de México, entre otras instituciones. Ha sido catedrático por 20 años en la UNPHU y en UNIBE. Ha sido distinguido con distintos premios entre los cuales se encuentra el $1^{\text {er }}$ premio del edificio para la Rosario Dominicana (1985); el $1^{\text {er }}$ premio para el Edificio Sede de la Suprema Corte de Justicia y la Procuraduría General de la República (1997); el 1er premio compartido para la sistematización de la plaza cívica del Centro de los Héroes (2004); y varios primeros en la Bienal de Arquitectura de Santo Domingo, de Arquitectura del Caribe y en la Bienal Nacional de Artes Visuales. Es autor de los libros HistorIAS PARA LA ConstRUcción DE LA Arquitectura Dominicana (2008), El Banco Central: 60 años de Arquitectura, Historia y Arte (2007) y Manual Biblográfico sobre la Arquitectura y el Urbanismo en el Gran Caribe, 1492-2005 (2008), entre otros. Es el editor de la revista Archivos de Arquitectura Antillana, de gran reconocimiento internacional. Preside el Grupo de Trabajo del DoCoMoMo Dominicano y coordina el Centro de Estudios de la Arquitectura, el Urbanismo y el Hábitat de FUNGLODE.
} 


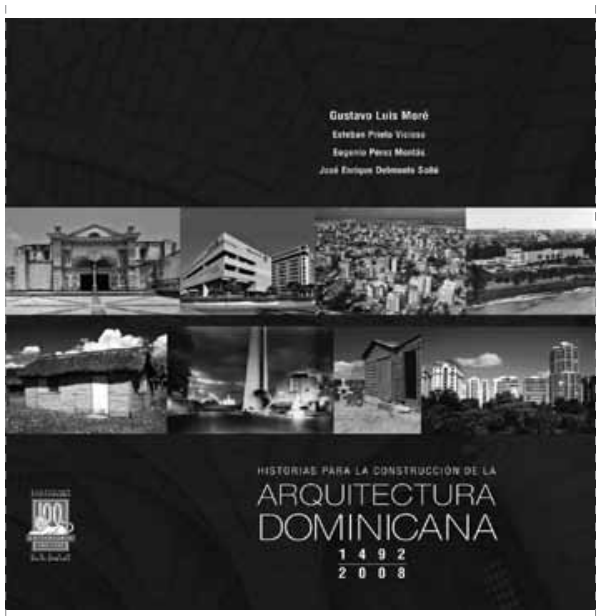

Portada del libro «Historias para la construcción de la arquitectura dominicana, 1492-2008», publicado en Santo Domingo con textos de los Arquitectos Eugenio Pérez Montás. Esteban Prieto Vicioso, José Enrique Delmonte y Gustavo Luis Moré. director del equipo.

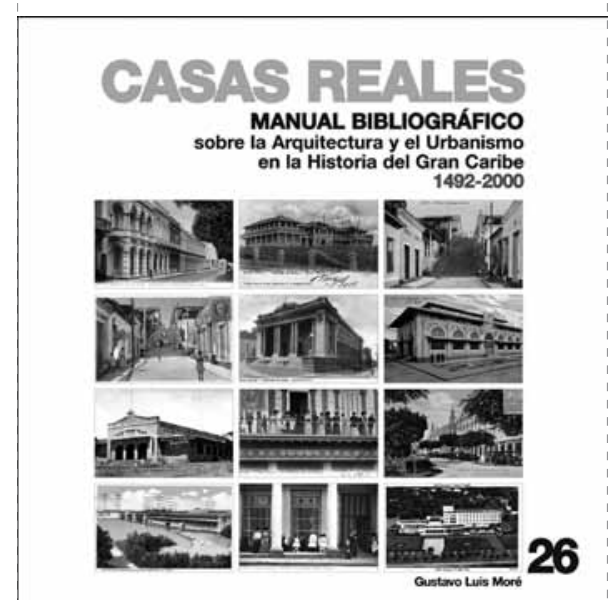

Portada del libro «Manual Bibliográfico sobre la Arquitectura y el Urbanismo del Gran Caribe, $1492-$ $2000 \%$, publicado como número 26 de la serie de la revista Casas Reales, en Santo Domingo, 2007 rasgos comunes -es inoportuno aquí hablar de invariantes- hacen posible una lectura panorámica del territorio, el devenir histórico de los pueblos que le configuran como entidad geográfica, ha determinado hechos tan disímiles que tornan inasible una visión homogénea de conjunto.

La verdadera historia se ha estado escribiendo durante un poco más de cinco siglos en la región hoy reconocida como el Gran Caribe; un territorio que se extiende desde las costas norteamericanas de la Florida, entre los seductores cayos del sur que casi orillan en La Habana, hasta el mar meridional de las tres exhuberantes Guyanas, atravesando un aparentemente infinito archipiélago de islas, grandes y pequeñas, que han sido tantas veces barajadas desde los poderes imperiales externos como estables en su identidad tropical. Un rosario de países describe el amplio arco que conecta la Isla de Trinidad con Venezuela por el Golfo de Paria, y define el periplo sobre la Tierra Firme de Colombia, Panamá, toda Centroamérica y el sorprendente Golfo de México desde el Yucatán hasta la Louisiana. Este itinerario recorre miles de kilómetros y atraviesa los más inverosímiles paisajes.

Hablar del Caribe exige una acto de reflexión cultural, una toma de consciencia que extralimita las categorías geográficas y obliga a identificar hechos labrados por la crónica común de muchos hombres, que, en el acto de construir sus sociedades, han adoptado hábitos impuestos por la aceptación de su inevitable realidad inmediata. Etnias diversas se han incorporado al teatro de la vida insular y costera de la región, hombres que han aprendido a querer mujeres de caderas oscuras, a tocar el tambor y a moverse sinuosamente, a arrancar raíces de la tierra y a cocinarlas con ingredientes y aromas parecidos, a domar la irascible naturaleza protegiéndose de los temibles vientos del este, de los volcanes implacables, de la tierra que danza sin aviso, y han concretado una serie de formas en el espacio, un desfile material de lugares capaces de alojarle y de, en el proceso, despertar emociones y llamar la atención de sus semejantes. Tal es la arquitectura en todo el planeta, tal es también la arquitectura del Gran Caribe.

Al hablar del $\mathrm{SAL}^{1}$, se hace conveniente establecer: ¿Dónde acaba el Caribe? ¿Dónde comienza Sudamérica? Las fronteras son como siempre, líneas inexistentes producto de la imaginación. El Gran Caribe llega hasta donde reinan el ron, la yuca y el plátano, el béisbol, y el tambor de toque cruzado, en clave, y termina allí donde empiezan el aguardiente, la papa y el maíz, el fútbol y el tambor derecho. ¿De qué otra forma acotarlo sino culturalmente? La perspectiva varía desde el punto de vista del observador: desde el archipiélago, la pacífica Panamá se entiende como un enclave del Caribe. Al preguntar en Panamá si se sienten caribeños, se nos responde: «el Caribe está del otro
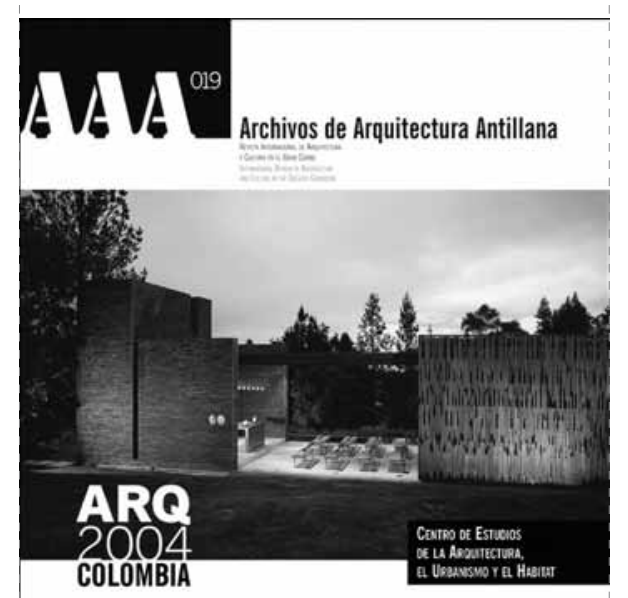

Portada de la edición AAA0019 dedicada a Colombia, de la revista dominicana Archivos de Arquitectura Antillana. Santo Domingo, 2004. lado, es la otra orilla...» Así, en el tiempo, la Arquitectura Caribeña ha sido el resultado de múltiples instancias políticas, de realidades polidimensionales. Aquel estrato de la colonia hispánica dominadora de las poblaciones indígenas originales, extendido sobre todo el territorio como una gigante cartografía en blanco, se fue desdibujando rápidamente, para ceder espacio a los africanos, franceses, ingleses, holandeses, daneses y portugueses, en un sucesivo e interminable ir y venir de caballos, telas, metales y ladrillos. Se construyó en el estilo de turno en el Imperio, nunca de forma clara, literal, siempre con un proyecto adaptado, marinado por las posibilidades de un mundo alternativo a Aquél, con minotauros de cabeza europea y cuerpo americano al frente de las paredes, sobre la tierra, bajo las bóvedas y al lado de las sombras verdinegras de una naturaleza ignota; con tal asamblea de hombres hubieron de aventurarse las primeras calles rectas, las primeras plazas geométricas; hubieron de levantarse las obras del Gótico y del Plateresco en el Santo Domingo ovandino y en el México monacal; las del Barroco en La Habana y en Veracruz; los anchísimos lienzos defensivos en Cartagena y en Portobelo; el Neoclásico de San Juan y San José; los frescos patios comunes a tantas y tantas islas, a tantos y tantos pueblos de criollos. En pocos años, las urgencias de la supervivencia y del comercio inventan casas y factorías, y barracones, y trapiches y muchas otras «maquinarias» sin estilo alguno, objetos dados a facilitar el 


\section{AMA \\ Archivos de Arquitectura Antillana}

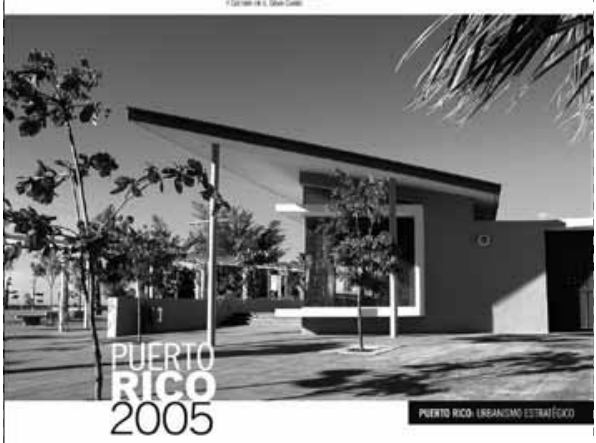

Portada de la edición AAA022 dedicada a Puerto Rico, de la revista dominicana Archivos de Arquitectura Antillana. Santo Domingo, 2006

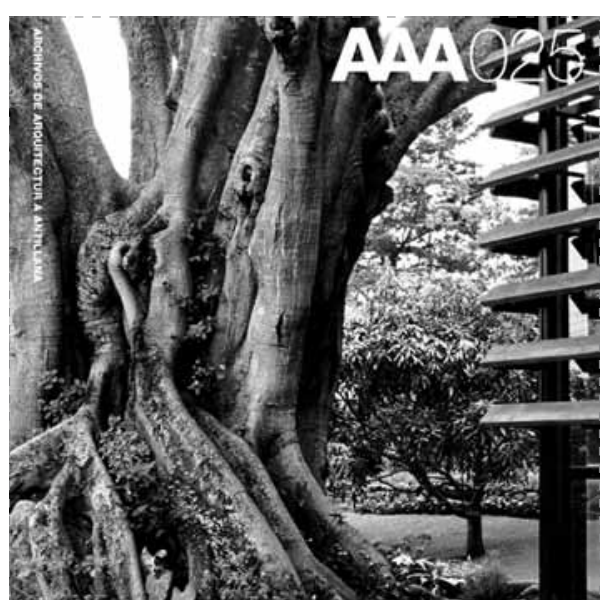

Portada de la edición AAA025 dedicada a Costa Rica, de la revista dominicana Archivos de Arquitectura Antillana, Santo Domingo, 2007.

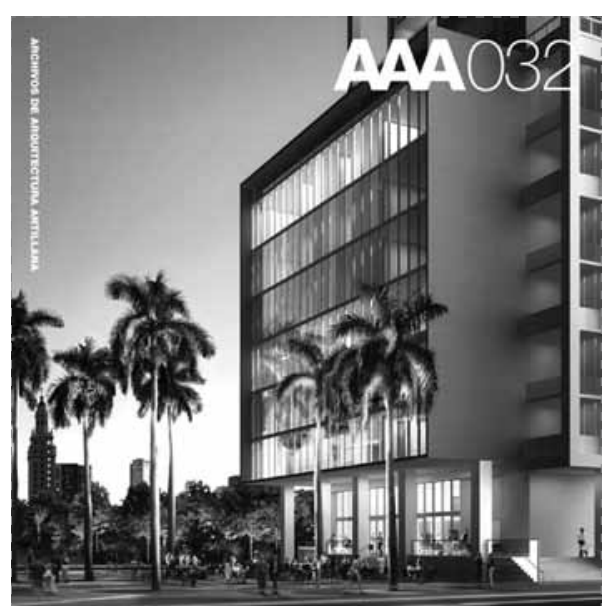

Portada de la edición AAA032 dedicada a Miami, de la revista dominicana Archivos de Arquitectura Antillana. Santo Domingo, 2008. trabajo de producción colonial instaurado en ese Caribe que, al decir de Benítez Rojo, «se repite» inagotablemente.

Los ingleses prefirieron la madera, el ladrillo de barro blanqueado que aparece en las great houses de Jamaica, en los edificios de la administración pública en Spanish Town; los franceses la piedra y la teja aplicada en sus grandes mansiones y sus alambiques de cobre para el intoxicante ron de caña; los holandeses pintaron los gabletes de sus edificios y landhuizen de ocres y luego de festivos colores, paleta que parece haberse incorporado a las construcciones populares de toda la región, originaria de quien sabe dónde; ya hacia fines del siglo xIx, con las revolucionarias iniciativas del comercio industrial, sobre caminos de hierro, entran las máquinas más veloces, más ruidosas, y la arquitectura cambia, se libera en galerías a veces metálicas articulándose a la ciudad en un segundo momento de ecléctica internacionalización: el Victoriano sustituye al Georgiano -desde Key West hasta Port-ofSpain, navegando por Puerto Plata, Ponce y las Islas Vírgenes-, el Gingerbread haitiano se apodera de Port-au-Prínce sustituyendo aquellas muraturas invencibles de la Citadelle del primer rey negro libre Christophe, los puertos del Caribe se transforman y crecen en la medida de que aumenta el intercambio. De nuevo, el lleva y trae del Caribe, esta vez, con otros bienes, con otras ilusiones.

El siglo xx fue pródigo en avances. Los ensayos de adaptación de la arquitectura de la modernidad varían de escenario en escenario, pero su presencia siempre obedece a protocolos reconocibles: jóvenes locales que acuden a universidades extranjeras y regresan portando consigo el germen del cambio; funcionarios gubernamentales que se designan en los territorios coloniales y ejecutan obras públicas significativas; aventureros inmigrantes que llegan por cualquier razón provenientes de estudios destacados en otros lares; universidades que transforman sus programas para dar cabida, no siempre de forma amable, a los nuevos teoremas de la redención social, formal y humana prometidos por los apóstoles de la blanca racionalidad. Cuba, con sus Batista, Borges, Romañach; Haití con su Mangonés; Dominicana con sus González, Caro, Pou Ricart; Puerto Rico con sus Klumb, Toro, Ferrer; Jamaica con Cheng; Martinica con Ali Tur; Guadalupe con Berthelot; Trinidad con Lewis y con Laird; Curazao con Smit; todos ellos pioneros de una transformación cultural aún no bien revelada por los estudiosos.

Desde esta óptica, hablar de un sólo Caribe es un equívoco, tan poco real como hablar de una sóla América Latina. Como señaló el maestro cubano Fernando Salinas, «la identidad no significa ser idénticos». Hoy propongo delinear todos los Caribes del Caribe. Cualquier aproximación crítica requiere de la lectura precisa de sus múltiples modos de producción, situados inevitablemente, en diversas circunstancias políticas, sociales y económicas. Valga citar los casos de países independientes desde el siglo xIx, como Venezuela o República Dominicana; otros desde 1962, como Jamaica y Trinidad; otros aún instalados como departamentos europeos de ultramar, como Martinica, Guadalupe o Curazao; otros de estatus un tanto ambiguo, como Puerto Rico; el caso de Cuba es, sin dudas una excepción, de diluida presencia en el ajedrez político regional, a pesar de sus recientes asociaciones internacionales.

A inicios de la segunda década del milenio, el Caribe visto desde el resto de América, es una controversial unidad erróneamente identificada con el mercado global del turismo, posicionada estratégicamente en esa cintura fronteriza todavía objeto de la explotación comercial de las grandes inversiones, y del dominio militar de los poderes globales. Hoy, otras arquitecturas dominan las ciudades, ahora hechas para convertirse en artículos de consumo, no necesariamente en bienes de uso cotidiano; ciudades tradicionalmente históricas asumen silentes la densificación, generalmente aleatoria de sus espacios patrimoniales, de sus territorios urbanos con potencial especulativo, fenómeno advertido en enclaves dispersos por toda la geografía: Miami, es el traslúcido objeto del deseo, Panamá el espejismo más virtual; Pero Cartagena, Santo Domingo, San José, San Salvador, atraviesan en la actualidad por fases de crecimiento extraordinariamente activas, producto de flujos económicos tan difusos como poderosamente fuertes.

Tal es el panorama en el que ha de actuarse hoy en el universo académico, a fin de proceder al registro crítico de la arquitectura y el urbanismo en la región del Gran Caribe. Este esfuerzo de síntesis requiere una actitud reductiva, de manera que las categorías de agrupación sean significativamente menores que los evidentemente complejos casos de análisis. La perspectiva de hoy trasciende los tradicionales esquemas de lectura basados en 


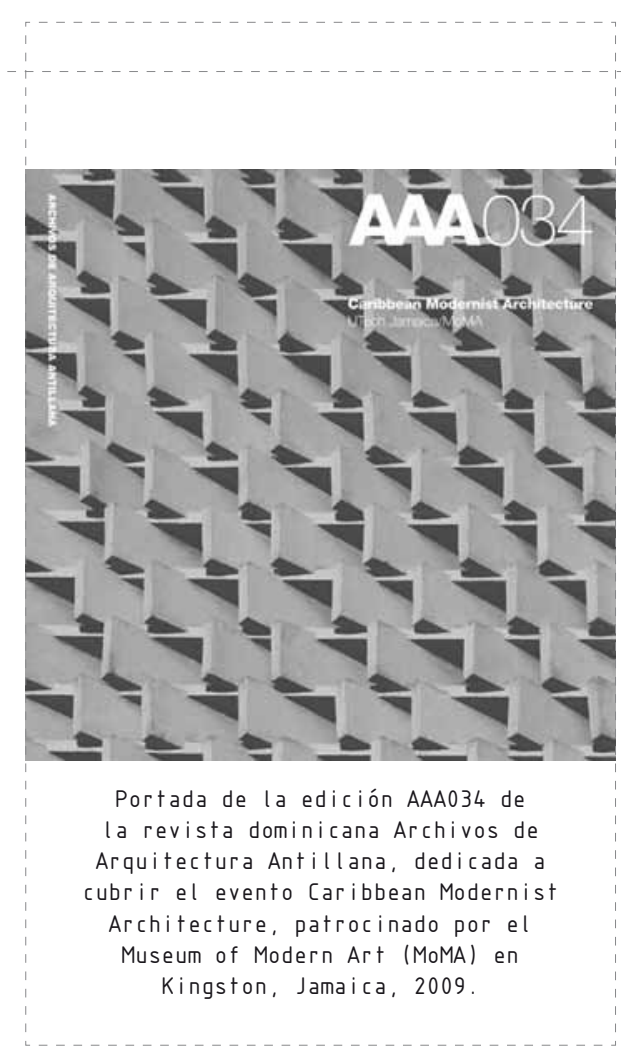

el análisis estilístico, si bien se hace inevitable garantizar la identificación de las asociaciones y afiliaciones al inventario de la arquitectura internacional valiéndonos de este. Más bien, hace falta construir un andamiaje conceptual capaz de estructurar las realidades paralelas más evidentes. En este sentido, se acusan algunas líneas comparativas de acción tales como:

- Las relaciones entre los sistemas económicos de producción y la arquitectura resultante; casos de estudio serían, por ejemplo las tres Antillas Mayores hispánicas, paradójicamente opuestas: el socialismo cubano, el capitalismo neocolonialista puertorriqueño, la independencia latinoamericanista dominicana.

- Las actuales afiliaciones imperiales europeas y los modos de producción regional; los casos a investigar serían, por ejemplo, países con normativas urbanas, códigos de diseño y mercados de abastecimiento europeos, como Guadalupe, Martinica (Francia), Curazao y Aruba (Holanda), y aquellos que se desempeñan en mercados liberados como los de América Central, Panamá o la República Dominicana.

- Los procesos de formación académica y su vinculación a la producción cultural y arquitectónica: casos como la única facultad de arquitectura existente para el Caribe inglés en Jamaica, o la necesidad de acceder a los sistemas académicos francés y holandés, para los territorios de cultura y lenguas correspondientes.

- El desarrollo local de la tecnología y de la industria de producción de materiales de construcción; los casos de República Dominicana, Colombia, Venezuela y México son extraordinariamente ricos frente a la escasa o inexistente producción local de las Antillas Menores.

En términos de la investigación sobre el patrimonio y la producción crítica sobre la arquitectura contemporánea, existen varios proyectos dignos de ser incluidos en una reseña como ésta:

1. Libros y volúmenes monográficos. La abundancia de la industria editorial en las naciones de mayor tamaño de la región es un hecho sabido de antemano. EE.UU., México y Colombia llevan la delantera en el sostenimiento de un sistema académico de investigadores institucionalizados en continua producción, aunque grandes avances son notorios en los últimos años en países como Puerto Rico, República Dominicana, Costa Rica y Panamá. Cuba ha mermado significativamente su otrora destacadísima producción editorial.

2. Revistas especializadas. La única revista de arquitectura de visión y alcance regional es Archivos de Arquitectura Antillana, publicada en Santo Domingo desde el año 1996. Varias publicaciones seriadas son producidas en Cuba (ARQUITECTURA) CuBA), Puerto Rico (la revista del CAAPPR), Jamaica (AxIS), Veracruz (ARQUITECTURA CON VaIVÉn de Hamaca), Barbados (Maco y Caribiean Architect), entre otras.

3. Tesis universitarias y estudios académicos Ya se ha mencionado la situación de los estudios de arquitectura en la región de las Antillas Menores, limitada al campus de la University of Jamaica. Las Mayores han perfilado una oferta mucho más abundante, sin hablar del caso de los países continentales, sobre todo Colombia, país en el que actualmente operan unas 25 escuelas, dos de ellas en la costa Caribe (Santa Marta, Barranquilla). Muchas de estas escuelas poseen programas de investigación y maestrías sobre las arquitecturas locales y en Santo Domingo se ha instaurado una Maestría en Arquitectura Caribeña que está reuniendo alumnos y profesores de toda la región, un programa ambicioso cuyos resultados comienzan a hacerse evidentes.
4. Los grupos de acción y resistencia que frecuentemente de manera informal generan el debate y la producción crítica en la región. El arquetipo es sin dudas el Grupo Nueva Arquitectura, fundado en Santo Domingo en 1989, que ha tenido una resonancia regional extraordinaria, fundando la itinerante Bienal de Arquitectura del Caribe, los Encuentros de Arquitectura y Urbanismo de las Antillas, entre muchas otras iniciativas de integración. También importante ha sido el Plan CARIMOS, una acción orientada originalmente al rescate y la valorización del patrimonio monumental interregional, canalizando repercusiones insospechadas. En la actualidad varios capítulos del DoCoMoMo Internacional operan en la región y han comenzado a desarrollar eventos comunes.

A nivel general, el asunto de la investigación en arquitectura y urbanismo en la región de Gran Caribe presenta serias distorsiones en su aproximación a los temas pendientes en nuestras respectivas agendas de desarrollo locales. No es suficiente producir la documentación históricamente certera y juiciosa en cuanto parte regional del universo de la historia del arte. Ya no es suficiente trabajar la Investigación por la Investigación misma, tanto como no vale el Arte por el Arte mismo. Habría que convertir la investigación en un instrumento al servicio del desarrollo, capaz de articular el uso del suelo y de los territorios patrimoniales dentro de las inicitivas de manejo públicas, asunto lamentablemente pendiente todavía. Hay que atar las iniciativas del buen gobierno y de la práctica profesional del diseño con la producción crítica e intelectual. De lo contrario, corremos el riesgo de automarginarnos y construir otro ghetto de especialistas, ajeno a las agudas problemáticas que actualmente aquejan a nuestras sociedades. Tal es el reto de la crítica arquitectónica contemporánea.

\section{Referencias bibliográficas}

AAA. Archivos de arquitectura antillana: revista internacional de arquitectura, urbanismo, historia y cultura en el Gran Caribe.

Moré, G. L. «Manual bibliográfico sobre la arquitectura y el urbanismo de la historia del Gran Caribe, 1492-2000».Programa Editorial del Plan CARIMOS, OEA, 2008.

Moré, G. L; Prieto, E; Pérez, E; Delmonte, G. E. «Historias para la construcción de la arquitectura dominicana (1492-2008)». Grupo León Giménez, República Dominicana, año 2009 